\title{
The influence of anti-TNF therapy upon incidence of keratinocyte skin cancer in patients with rheumatoid arthritis: longitudinal results from the British Society for Rheumatology Biologics Register
}

\author{
Louise K Mercer, ${ }^{1}$ Adele C Green, 2,3 James B Galloway, ${ }^{1}$ Rebecca Davies, ${ }^{1}$ Mark Lunt, ${ }^{1}$ \\ William G Dixon, ${ }^{1}$ Kath D Watson, ${ }^{1}$ British Society for Rheumatology Biologics Register \\ Control Centre Consortium* ${ }^{*}$ Deborah PM Symmons, ${ }^{1}$ Kimme L Hyrich, ${ }^{1}$ \\ on behalf of the British Society for Rheumatology Biologics Register
}

\begin{abstract}
- Additional supplementary materials are published online only. To view the files please visit the journal online (http://ard.bmj.com/ content/71/6.toc).

${ }^{1}$ Arthritis Research UK Epidemiology Unit, The University of Manchester, Manchester Academic Health Science Centre, Manchester, UK ${ }^{2}$ School of Translational Medicine, The University of Manchester, Manchester Academic Health Science Centre, Manchester, UK

${ }^{3}$ Cancer and Population Studies Group, Queensland Institute of Medical Research, Brisbane, Australia

${ }^{4}$ British Society for Rheumatology, London, UK
\end{abstract}

*For list of member institutions of the British Society for Rheumatology Biologics Register Control Centre Consortium see end of paper.

\section{Correspondence to} Dr Kimme L Hyrich, Arthritis Research UK Epidemiology Unit, Stopford Building, The University of Manchester, Manchester Academic Health Science Centre, Oxford Road, Manchester, M13 9PT, UK: Kimme.hyrich@manchester.ac.uk

Received 25 October 2011 Accepted 29 November 2011 Published Online First 12 January 2012

\begin{abstract}
Objectives To compare the risk of keratinoctye skin cancer (basal cell carcinoma (BCC) and squamous cell carcinoma (SCC)) in patients treated for rheumatoid arthritis (RA) compared with the general population, and to determine whether anti-tumour necrosis factor (TNF) therapy exacerbates this risk.
\end{abstract}

Methods Patients with RA enrolled in the British Society for Rheumatology Biologics Register, a prospective national cohort established in 2001 to monitor the safety of anti-TNF, were followed until 2008. 11881 patients treated with anti-TNF were compared with 3629 patients receiving non-biological disease-modifying antirheumatic drugs (nbDMARD). Standardised incidence ratios (SIR) were calculated for each cohort and rates between cohorts were compared using Cox proportional HR, adjusted using inverse probability of treatment weighting.

Results SIR for skin cancer was increased in both cohorts compared with the English population: SIR 1.72 $(95 \% \mathrm{Cl} 1.43$ to 2.04$)$ anti-TNF; 1.83 (95\% $\mathrm{Cl} 1.30$ to 2.50) nbDMARD only. In patients without previous skin cancer, BCC incidence per 100000 patient-years was 342 (95\% Cl 290 to 402) after anti-TNF and 407 (95\% Cl 288 to 558) after nbDMARD. HR after anti-TNF adjusted for treatment weighting was $0.95(95 \% \mathrm{Cl} 0.53$ to 1.71$)$. SCC incidence per 100000 patient-years: anti-TNF 53 (95\% Cl 33 to 79 ); nbDMARD 43 (95\% Cl 12 to 110); adjusted HR 1.16 (95\% Cl 0.35 to 3.84$)$.

Conclusions Skin cancers were increased among treated patients with RA. No evidence was found that anti-TNF therapy exacerbates the risk of BCC or SCC but this cannot be excluded. Patients with RA should use sun protection and be monitored for skin cancer.

Keratinocytic skin cancers, referred to here as 'skin cancer', mostly comprise basal cell carcinoma (BCC) (approximately $75 \%)^{1}$ and squamous cell carcinoma (SCC). These are the most commonly diagnosed cancers in the USA and UK. ${ }^{2}{ }^{3}$ The annual incidence of skin cancer varies by latitude, age and sex. In New Hampshire, USA (1993-4) the age-adjusted rates per 100000 population for BCC were 310 in men and 166 in women, ${ }^{4}$ whereas in Arizona, USA (1996), corresponding rates for BCC were 936 and 497 in men and women, respectively. ${ }^{5}$
Some studies have found an increased risk of skin cancer in patients treated for rheumatoid arthritis (RA), ${ }^{67}$ whereas other studies have not. ${ }^{89}$ A Swedish study found no increased risk of SCC in a cohort of patients with early RA but did find a $66 \%$ increased risk in patients with prevalent RA. ${ }^{10}$ The standardised incidence ratio (SIR) for SCC increased with the duration of follow-up, suggesting the risk may be related to cumulative RA duration and/or immunosuppressive therapy.

Anti-tumour necrosis factor (TNF) drugs have proved highly successful in treating RA. Five antiTNF drugs are approved for use in North America and Europe; etanercept, adalimumab, infliximab, golimumab and certolizumab pegol. The introduction of TNF inhibitors has been accompanied by concerns regarding safety, and in particular the risk of malignancy. ${ }^{11}$ As the most common malignancies are skin cancers, then if TNF inhibition does have an effect on the risk of malignancy, one might predict that the signal would be seen first in the skin. Data from clinical trials and observational studies in North America and Sweden have reported an increased risk of skin cancer with anti-TNF compared with the general population and untreated patients with RA. ${ }^{10} 12-14$

The first aim of this study was to compare the incidence of skin cancer in anti-TNF-exposed and biological-naive patients with rates in the UK general population, using data from a national UK cohort of people with RA. The second aim was to explore the additional influence, if any, of anti-TNF therapy on the incidence of BCC and SCC by comparing incidence rates in these two cohorts.

\section{METHODS \\ Patients}

The subjects' written consent was obtained. The study was approved by the North-West Multicentre Research Ethics Committee. Subjects were participants in the British Society for Rheumatology Biologics Register (BSRBR), a national prospective observational study established in 2001 to monitor the long-term safety of biological therapy in RA. UK national guidelines recommend that anti-TNF is restricted to patients 
with active disease (defined as 28 joint disease activity score (DAS28) >5.1) despite treatment with at least two non-biological disease-modifying antirheumatic drugs (nbDMARD), one of which should be methotrexate. ${ }^{15}$ These guidelines recommend against the use of anti-TNF in patients with earlier malignancy within the previous decade. During the time period studied in this analysis, three anti-TNF agents were available in the UK: infliximab, etanercept and adalimumab. A comparison cohort of biological-naive RA patients was recruited in parallel and followed in an identical manner to the anti-TNF cohort. ${ }^{16}$ These patients had active disease at recruitment (DAS28 24.2 ) despite current treatment with at least one nbDMARD.

Patients were eligible if they had a physician diagnosis of RA and at least one returned rheumatologist follow-up questionnaire before 31 December 2008. The anti-TNF cohort was restricted to patients who received an anti-TNF as their first biological therapy and who registered with the BSRBR within 6 months of starting treatment. Patient-years of follow-up time were calculated from the date of starting an anti-TNF, or the date of registration with the BSRBR for the nbDMARD cohort. Follow-up was censored at the date of the most recently received hospital follow-up form before 31 December 2008 or death, if this came first. Patients were not censored at the point of skin cancer diagnosis, as they could be diagnosed with more than one skin cancer during follow-up. While only cancers diagnosed before 31 December 2008 were included in the analyses, we accepted all reports of such cancers up to 30 September 2010, to allow for any lag in cancer reporting to the BSRBR. Patients in the nbDMARD cohort who subsequently started an anti-TNF contributed follow-up time up until the date of the first dose of anti-TNF. As anti-TNF therapy may continue to influence the risk of skin cancer after its cessation, followup time after stopping anti-TNF was included in the analysis. Patients were censored from the analysis at the point of starting a biological disease-modifying antirheumatic drug other than anti-TNF.

\section{Baseline assessment}

Baseline data included demographics, disease duration, disease activity, current and past nbDMARD, comorbidities and smoking history. Patients completed a Stanford health assessment questionnaire (HAQ) $)^{17}$ to indicate their level of physical disability. Previous malignancies, including skin cancers, were reported directly by the National Health Service Information Centre (NHS-IC) who link with the Office for National Statistics, which has been shown to register $90 \%$ of malignancies diagnosed in the UK accurately. ${ }^{18}$ Registration of skin cancer is less complete as the UK Association of Cancer Registries suggests that only the first BCC or SCC per patient should be recorded. ${ }^{19}$

\section{Follow-up}

Changes to therapy were reported on rheumatologist questionnaires 6-monthly for 3 years, then annually thereafter. Data on adverse events were captured in three ways: from rheumatologist questionnaires; from 6-monthly patient diaries completed for 3 years; and by flagging with NHS-IC, which reported malignancies using the 10th edition of the International Classification of Diseases (ICD-10) and the International Classification of Diseases for Oncology, which distinguishes between BCC and SCC.

Additional information (including histology) was sought from physicians for all incident skin cancers. Two physicians (LKM

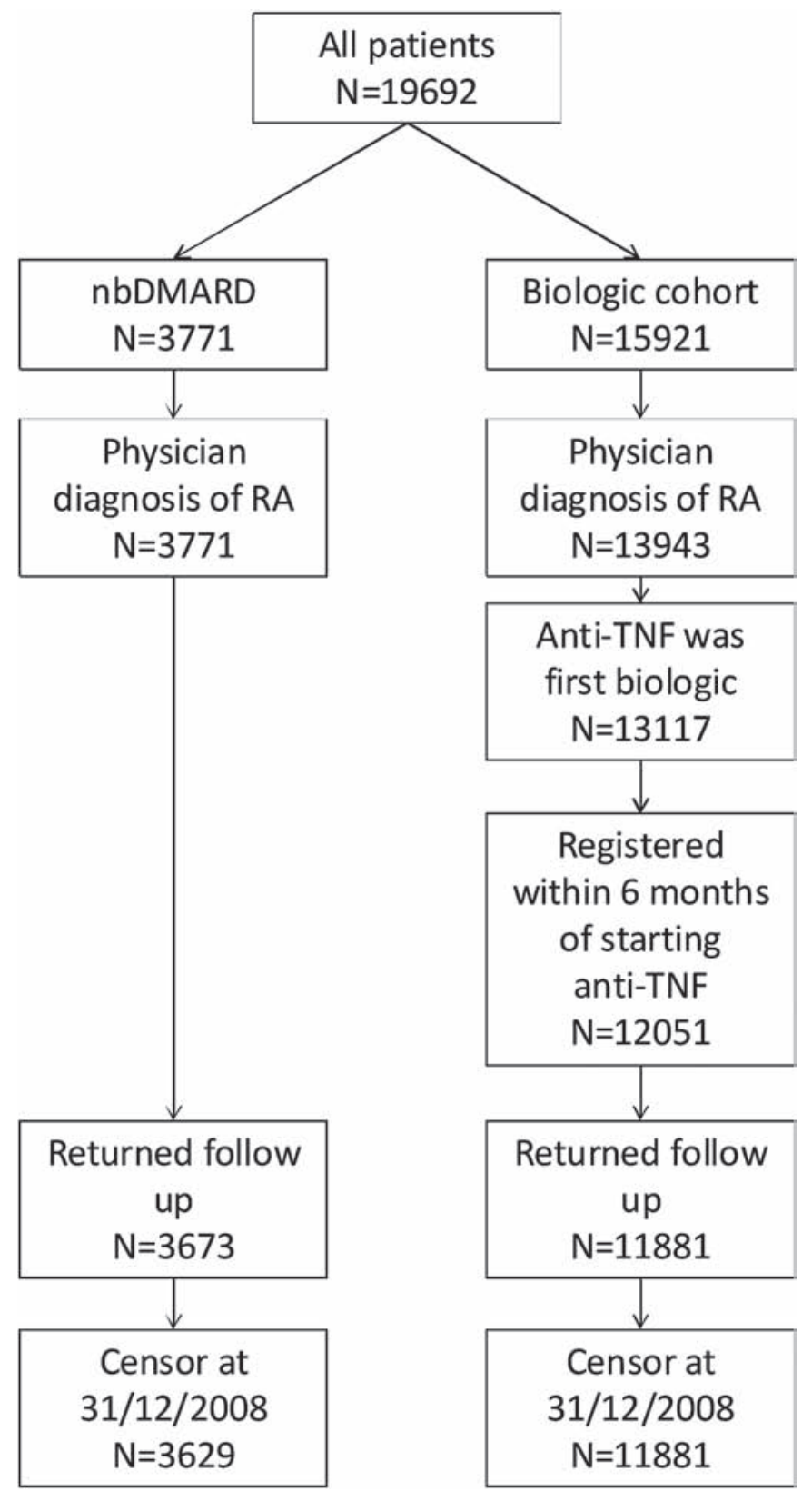

Figure 1 Flowchart showing selection of patients for the analysis. nbDMARD, non-biological disease-modifying antirheumatic drug; RA, rheumatoid arthritis; TNF, tumour necrosis factor.

and JBG) independently verified all reports of skin cancers. Skin cancers were verified if they were reported by NHS-IC or histology confirmed the diagnosis. Bowen's disease and known preexisting skin cancers were excluded.

\section{Statistical analysis}

The risk of skin cancer in RA patients living in England was compared with the English general population. Age and genderstandardised rates of skin cancer for the entire UK population were not available and numbers of skin cancers occurring in BSRBR patients living in Northern Ireland, Scotland and Wales were insufficient for individual analyses. For England, SIR were calculated separately for the nbDMARD and anti-TNF cohorts. English population rates of skin cancer (excluding melanoma) from 2003 to 2008 were used. To ensure consistency between observed and expected rates we included only those cancers that 
Table 1 Baseline characteristics of patients

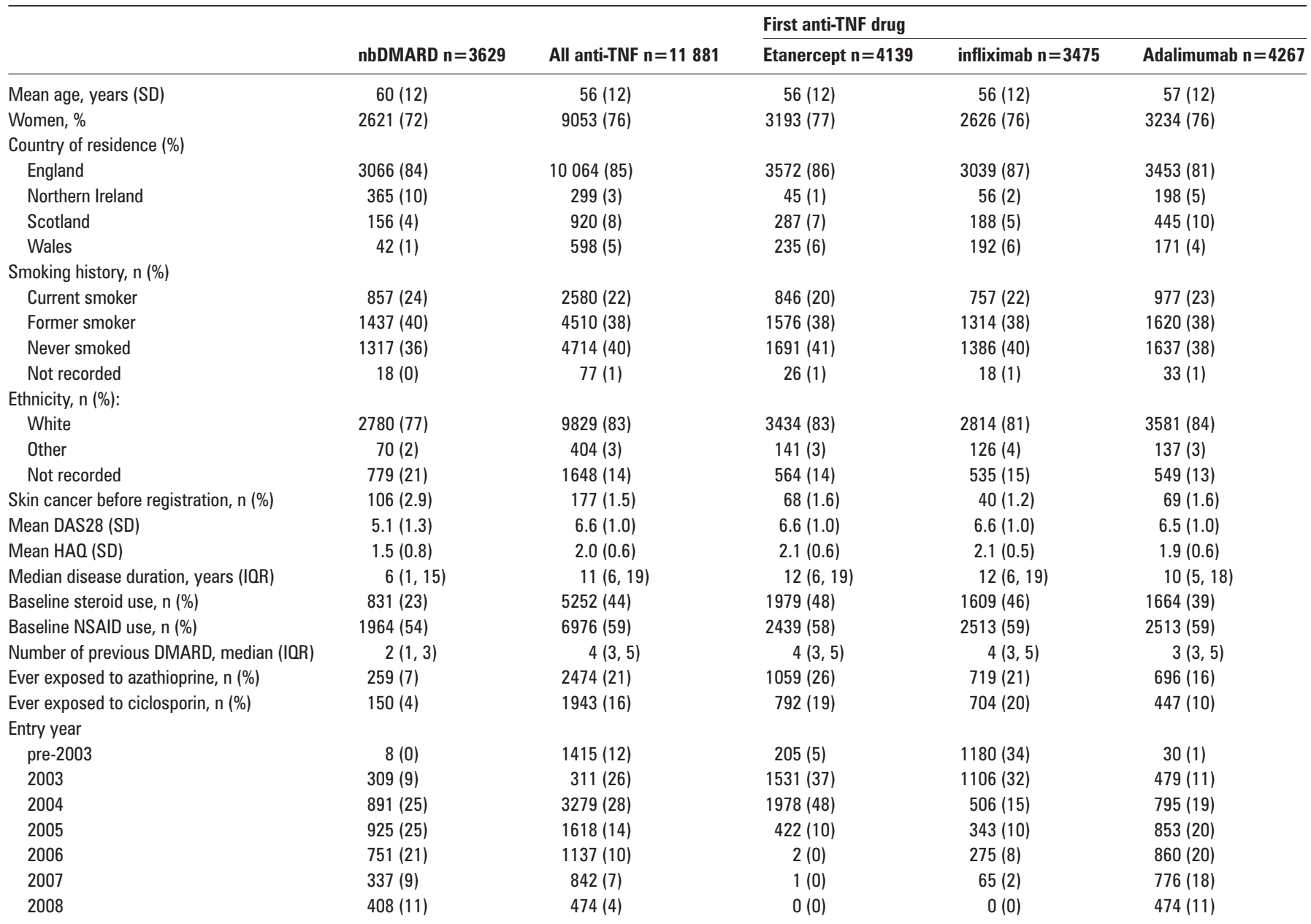

DAS28, disease activity score in 28 joints; HAQ, health assessment questionnaire; nbDMARD, non-biological disease-modifying antirheumatic drug; NSAID, non-steroidal anti-inflammatory drug; TNF, tumour necrosis factor.

had been reported to us from NHS-IC. Patients with a history of skin cancer before registration were included in this analysis because such patients could not be excluded from the population rates.

Risk comparisons were made between the anti-TNF and nbDMARD cohorts, first limited to patients without a previous history of skin cancer (BCC, SCC, melanoma or other skin cancer) at the time they joined the BSRBR. Rates of BCC and SCC are presented as total events per 100000 patient-years with $95 \%$ CI. Survival analysis using Cox regression was used to compare the difference in risk between the anti-TNF and nbDMARD cohorts. Potential confounders were identified a priori and comprised potential risk factors for skin cancer (age, sex, smoking and previous exposure to ciclosporin and azathioprine), non-steroidal anti-inflammatory drugs (NSAID), as these have been shown to protect against skin cancer, ${ }^{20}$ and differences in baseline characteristics (DAS28, HAO, disease duration, baseline steroid use and year of entry to the study). Adjustment for these covariates was performed by calculating a propensity score, which reflected the likelihood that an individual patient was prescribed an anti-TNF given their known characteristics. The inverse of this score (or one minus the inverse for the nbDMARD cohort) was used as a weight in the Cox regression analysis. Missing baseline data were replaced using multiple imputations (see supplementary methods, available online only). The assumption of proportionality was tested using Schoenfeld residuals and met.
Table 2 Standardised incidence ratios of NHS-IC reported skin cancers in England compared with England population 2003-8 (including patients with previous skin cancer)

\begin{tabular}{|c|c|c|c|}
\hline & \multicolumn{3}{|c|}{ Skin cancer (excluding melanoma) ICD-10 C44 } \\
\hline & No & Expected & $\operatorname{SIR}(95 \% \mathrm{CI})$ \\
\hline nbDMARD & 39 & 21.34 & $1.83(1.30$ to 2.50$)$ \\
\hline Men & 15 & 8.97 & 1.67 (0.94 to 2.76$)$ \\
\hline Women & 24 & 12.38 & 1.94 (1.24 to 2.89$)$ \\
\hline Anti-TNF & 126 & 73.44 & 1.72 (1.43 to 2.04$)$ \\
\hline Men & 48 & 23.79 & 2.02 (1.49 to 2.68$)$ \\
\hline Women & 78 & 49.65 & $1.57(1.24$ to 1.96$)$ \\
\hline
\end{tabular}

ICD-10, International Classification of Diseases, 10th edition; nbDMARD, non-biological disease-modifying antirheumatic drug; NHS-IC, National Health Service Information Centre; SIR, standardised incidence ratio; TNF, tumour necrosis factor.

Individual comparisons of the anti-TNF agents with the nbDMARD cohort were made for BCC. A BCC was attributed to the anti-TNF prescribed at the time of skin cancer diagnosis, or to the most recently received anti-TNF. Three sensitivity analyses on the rate of BCC were planned in advance. First, an analysis was limited to the first BCC per patient only. Second, to investigate the effects of any possible screening bias, the first year of follow-up was excluded from the analysis. Third, an analysis was performed limited to patients with a previous history of reported BCC. No drug-specific analyses were performed for SCC. As SCC are rare in the UK population aged 
Table 3 Skin cancer reported in patients without previous history of skin cancer

\begin{tabular}{lccccc}
\hline & nbDMARD & Anti-TNF & Etanercept & Infliximab & Adalimumab \\
\hline Number ever exposed to drug during follow-up & 3523 & 11704 & 5086 & 3663 & 5035 \\
Patients with cancer & 38 & 139 & 54 & 49 & 36 \\
Cancers & 43 & 176 & 67 & 67 & 42 \\
Patients with multiple cancers (\%) & $3(8)$ & $27(19)$ & $10(18)$ & $13(27)$ & $4(11)$ \\
BCC (\%) & $38(88)$ & $150(85)$ & $57(85)$ & $59(88)$ & $34(81)$ \\
SCC (\%) & $4(9)$ & $23(13)$ & $9(13)$ & $8(12)$ & $6(14)$ \\
Basosquamous cell carcinoma (\%) & $0(0)$ & $1(1)$ & $0(0)$ & $0(0)$ & $1(2)$ \\
Dermatofibrosarcoma protuberans & $0(0)$ & $1(1)$ & $1(1)$ & $0(0)$ & $0(0)$ \\
Unclassified skin cancer (\%) & $1(2)$ & $1(1)$ & $0(0)$ & $0(0)$ & $1(2)$ \\
First skin cancer reported by (\%): & & & & & \\
$\quad$ NHS-IC & $36(95)$ & $121(87)$ & $49(91)$ & $38(78)$ & $34(94)$ \\
Physician and/or patient & $17(45)$ & $85(61)$ & $35(65)$ & $33(67)$ & $17(47)$ \\
\hline
\end{tabular}

BCC, basal cell carcinoma; nbDMARD, non-biological disease-modifying antirheumatic drug; NF, tumour necrosis factor; NHS-IC National Health Service Information Centre; SCC, squamous cell carcinoma; TNF, tumour necrosis factor.

under 55 years, ${ }^{21} 22$ a sensitivity analysis for SCC restricted to patients aged over 55 years was performed. In addition to the main 'ever exposed to anti-TNF' analyses, rates of BCC and SCC in patients actively receiving anti-TNF, or within 90 days of stopping ('on drug'), were compared with nbDMARD. All analyses were conducted using Stata version 10.1.

\section{RESULTS}

Three thousand six hundred and twenty-nine nbDMARD and 11881 anti-TNF patients were included (figure 1). The antiTNF cohort was younger and comprised more women (table 1). Eighty-five per cent of patients were living in England. A known previous history of skin cancer was lower in the anti-TNF cohort $(1.5 \%$ vs $2.9 \%)$. The anti-TNF cohort had more severe disease of longer duration. Two hundred and thirty-four patients enrolled in the nbDMARD cohort subsequently registered with the antiTNF cohort and so contributed follow-up time to both. Twentyfour patients were censored from nbDMARD and 1327 from anti-TNF when starting other biological therapy.

\section{Risk of skin cancer in patients with RA compared with the general population for England}

The SIR for overall skin cancer was similar and significantly increased in both treatment cohorts; SIR 1.72 (95\% CI 1.43 to 2.04 ) anti-TNF; 1.83 (95\% CI 1.30 to 2.50$)$ nbDMARD (table 2). In the anti-TNF cohort, the SIR was higher for men than women (2.02, 95\% CI 1.49 to 2.68 vs $1.57,95 \%$ CI 1.24 to 1.96$)$ whereas in the nbDMARD-only cohort the SIR was higher in women.

\section{Risk of skin cancer for anti-TNF in patients without known past history of skin cancer}

This analysis included 14993 patients. One thousand nine hundred and twenty-eight patients received more than one anti-TNF drug during follow-up. Two hundred and nineteen skin cancers were diagnosed during 53140 patient-years of follow-up (176 in the anti-TNF cohort and 43 in the nbDMARD cohort (tables $3,4)$ ). For anti-TNF, $19 \%$ of patients with incident skin cancer had multiple or recurrent lesions compared with $8 \%$ of the nbDMARD-only cohort $\left(\chi^{2} p=0.093\right)$. In the anti-TNF cohort, 121 (87\%) first skin cancers were reported via NHS-IC compared with $36(95 \%)$ in the nbDMARD cohort $\left(\chi^{2} \mathrm{p}=0.185\right)$. One hundred and twenty-four $(70 \%)$ of the skin cancers in the anti-TNF cohort occurred while the patient was receiving an anti-TNF drug. All skin cancers occurred in white patients.

\section{Basal cell carcinoma}

The unadjusted risk of BCC for anti-TNF compared with nbDMARD only was 0.84 (95\% CI 0.58 to 1.20 ; table 4). Age, male gender, disease duration and previous exposure to azathioprine or ciclosporin were associated with a risk of BCC in univariate analysis (see supplementary table S1, available online only). Current smokers and patients taking NSAID at baseline were at reduced risk of BCC in univariate analysis. The fully adjusted HR for anti-TNF was 0.95 (95\% CI 0.53 to 1.71). Analysis stratified by anti-TNF drug found a higher risk for infliximab than for the other two anti-TNF, although this was not statistically significant. The observed risk for infliximab was further attenuated in a sensitivity analysis limited to first BCC. After excluding the first year of follow-up, the HR for anti-TNF was not significantly increased $(1.18,95 \%$ CI 0.60 to 2.32$)$.

\section{Squamous cell carcinoma}

The unadjusted HR for anti-TNF was 0.93 (95\% CI 0.32 to 2.76 ; table 4). After adjustment for treatment weighting there was no association between anti-TNF and SCC (HR 1.16, 95\% CI 0.35 to 3.84$)$. When restricted to patients aged over 55 years the HR for anti-TNF was 1.32 (95\% CI 0.33 to 5.32$)$.

\section{Risk of BCC in patients with known previous skin cancer}

Two hundred and eighty-three patients had a history of skin cancer before entering the study (177 anti-TNF cohort; $106 \mathrm{nbD}$ MARD cohort). The crude incidence rate of BCC was 10-fold higher in this cohort compared with those without previous cancer, and higher in the nbDMARD cohort compared with anti-TNF (7982 per 100000 patient-years vs 4623 per 100000 patient-years). The adjusted risk for anti-TNF was 0.70 (95\% CI 0.26 to 1.94$)$.

\section{DISCUSSION}

The BSRBR is the largest biologicals register of its kind, with detailed follow-up for a median of 4 years and up to 8 years. The study has the unique advantage of the flagging of patients with the NHS-IC that provided details of all registered previous and incident BCC and SCC. The results show that compared with the general English population, the overall risk of skin cancer was increased in patients treated for RA, regardless of treatment history.

Our findings add to the results of several observational studies of RA. ${ }^{6-8} 1023-25$ A Danish study found a $40 \%$ increased risk of SCC and 30\% relative increase in BCC. ${ }^{7}$ However, this finding has not been consistent, and studies from Finland, Sweden and 
Table 4 Risk of skin cancer for anti-TNF therapy in patients with RA without previous history of skin cancer

\begin{tabular}{|c|c|c|c|c|c|c|c|}
\hline & \multicolumn{5}{|l|}{$\mathrm{BCC}$} & \multicolumn{2}{|l|}{ SCC } \\
\hline & nbDMARD & Anti-TNF & Etanercept & Infliximab & Adalimumab & nbDMARD & Anti-TNF \\
\hline $\begin{array}{l}\text { Number ever exposed to } \\
\text { drug during follow-up }\end{array}$ & 3523 & 11704 & 5086 & 3663 & 5035 & 3523 & 11704 \\
\hline $\begin{array}{l}\text { Median exposure time } \\
\text { per subject (years) }\end{array}$ & 2.65 & 4.01 & 3.70 & 2.70 & 2.05 & 2.65 & 4.01 \\
\hline Patients with cancer & 34 & 121 & 47 & 45 & 29 & 4 & 19 \\
\hline Unadjusted HR (95\% Cl) & Ref & 0.84 (0.58 to 1.20 ) & 0.71 (0.47 to 1.08 ) & 1.25 (0.83 to 1.89 ) & 0.64 (0.40 to 1.02 ) & Ref & 0.93 (0.32 to 2.76$)$ \\
\hline Age and gender $\mathrm{HR}(95 \% \mathrm{CI})$ & Ref & $1.20(0.83$ to 1.73$)$ & 1.07 (0.70 to 1.63 ) & 1.73 (1.14 to 2.62$)$ & 0.89 (0.56 to 1.42$)$ & Ref & $1.79(0.59$ to 5.41$)$ \\
\hline aHR (95\% Cl) & Ref & 0.95 (0.53 to 1.71$)$ & 0.80 (0.44 to 1.47 ) & 1.47 (0.76 to 2.85$)$ & $0.73(0.37$ to 1.46$)$ & Ref & $1.16(0.35$ to 3.84$)$ \\
\hline $\begin{array}{l}\text { First cancer per subject } \\
\text { incident rate/100 } 000 \\
\text { patient-years }(95 \% \mathrm{Cl})\end{array}$ & 364 (252 to 509$)$ & 276 (229 to 330$)$ & 246 (181 to 328 ) & 376 (273 to 505$)$ & 231 (156 to 330) & & \\
\hline On drug analysis aHR (95\% Cl) & Ref & 0.88 (0.48 to 1.60$)$ & 0.68 (0.36 to 1.29$)$ & 1.24 (0.63 to 2.46$)$ & 0.68 (0.32 to 1.42 ) & Ref & 0.96 (0.28 to 3.32$)$ \\
\hline
\end{tabular}

aHR, hazard ratio adjusted for inverse probability of treatment weighting; BCC,basal cell carcinoma; nbDMARD, non-biological disease-modifying antirheumatic drug; RA, rheumatoid arthritis; Ref, referent; SCC, squamous cell carcinoma; TNF, tumour necrosis factor.

Scotland have not reported an increased risk. ${ }^{6} 823$ An explanation for the increased skin cancer in the BSRBR cohort may be that that study comprised individuals with long-standing RA with high disease activity at baseline, all of whom have been exposed to disease-modifying antirheumatic drugs. The results may not be generalisable to patients with mild disease.

Other reasons for differing results may also include variations in definitions of skin cancer (with melanoma, SCC, BCC and other cancers being variably combined) and different methods of case ascertainment. We used English population data from 2003 to 2008, during which time it has been acknowledged that the reporting of skin cancers to the national registry was incomplete. ${ }^{26}$ We only included cancers that were reported to us via NHS-IC in this analysis to account for this, meaning any underreporting would occur equally and introduce no bias. However, we found reporting of first skin cancers via NHS-IC to be similar in both cohorts; nbDMARD only $95 \%$ and anti-TNF $87 \%$. In comparison, fewer cancers were physician reported, which may reflect the fact that most skin cancers are diagnosed in primary care or dermatology clinics and may not have been recorded in rheumatology casenotes.

In patients without reported previous skin cancer we found that the addition of anti-TNF did not exacerbate their risk of skin cancer when compared with nbDMARD alone with follow-up for several years. This is in keeping with a metaanalysis of randomised controlled trials of anti-TNF in RA. ${ }^{27}$ Conversely, another randomised controlled trial meta-analysis of anti-TNF across all indications found a twofold increased risk of skin cancer. ${ }^{12}$ In addition, an earlier analysis of BSRBR data, published in abstract form, ${ }^{28}$ reported a possible signal for an increased risk of skin cancer. This earlier analysis relied more heavily on cancer reporting by the physicians as we only allowed a 6-month time lag for NHS-IC reporting. This may have increased the effect of reporting bias whereby physicians preferentially reported adverse events in patients treated with biological agents, perhaps demonstrated by the lower proportion of cases reported by the physician in the nbDMARD cohort. The updated analysis presented here allows for up to a 21-month lag in reporting by the NHS-IC and more complete case ascertainment by this source, as regional cancer registries are allowed up to 18 months to report cancers to the Office for National Statistics. ${ }^{29}$ As this source is blind to the treatment arm of patients, it is less susceptible to bias, although a general surveillance bias among patients treated with anti-TNF therapies cannot be completely excluded.

In this study we also compared the risk of skin cancer between individual drugs. There was a suggestion that the incidence of BCC was higher for infliximab compared with nbDMARD than for etanercept or adalimumab, although this finding did not reach statistical significance. A US study found an increased risk of skin cancer for infliximab (OR 1.7, 95\% CI 1.3 to 2.2) but not for etanercept $(1.2,95 \%$ CI 1.0 to 1.5$)$ or adalimumab $(0.9,95 \%$ CI 0.5 to 1.8$) .{ }^{13}$ That study was unable to distinguish between BCC and SCC, which makes interpretation difficult given their different pathobiologies. Furthermore, in that study cases of malignancy were patient reported and not always histologically confirmed. ${ }^{13}$

In our study, the higher rate for infliximab may be partly explained by a small number of infliximab patients being diagnosed with multiple BCC, as the HR for infliximab was attenuated when the analysis was limited to first skin cancer. Surveillance bias may also explain this finding, as infliximab-treated patients were assessed every 8 weeks before each infliximab infusion: a hypothesis further strengthened by the higher rate of physicianreported events in patients receiving infliximab.

An important consideration of this study relates to the SCC risk in the anti-TNF cohort. Given that SCC is uncommon in the UK, this study may lack power to detect an important increased risk following anti-TNF. An indication that this may be case was that the HR for anti-TNF was higher when restricted to patients aged over 55 years. It is also important to bear in mind that in the UK, a history of previous malignancy is listed as a contraindication to anti-TNF. The finding of a 10 -fold higher incidence of BCC in patients with previous BCC was in keeping with data from the general population. ${ }^{30}$

As with any observational study, it is important to acknowledge the potential for unmeasured confounding. We have collected detailed patient data at baseline for which we have made 
adjustments. We lack data on factors such as exposure to ultraviolet light or skin type.

\section{CONCLUSIONS}

Despite the difficulties in determining valid population rates of skin cancer, the findings from this study add weight to the evidence that skin cancers are increased among treated patients with RA, although it cannot support the hypothesis that anti-TNF therapy specifically increases this risk of BCC. An increased risk of SCC cannot be excluded. Recommendations for skin cancer prevention in the general population, such as sun avoidance, apply to patients with RA. These data support the regular monitoring of patients with RA for unusual or new skin lesions.

Acknowledgements The authors acknowledge the enthusiastic collaboration of all consultant rheumatologists and their specialist nurses in the UK in providing the data. In addition, the authors acknowledge the support from Dr lan Griffiths (past) and Professor David Isenberg (current) chairs of the BSRBR management committee, Professor Gabriel Panayi, Professor David GI Scott, Dr Andrew Bamji and Professor Deborah Bax, presidents of the BSR during the period of data collection, for their active role in enabling the register to undertake its tasks and to Samantha Peters (CEO of the BSR), Mervyn Hogg, Nia Taylor and members of the BSRBR scientific steering committee. The authors also acknowledge the seminal role of the BSR clinical affairs committee for establishing national biological guidelines and recommendations for such a register. Finally the authors would like to acknowledge the substantial contribution of Andy Tracey, Katie McGrother and Dr Mark Lunt in database design and manipulation and Professor Alan Silman in his earlier role as a principal investigator of the BSRBR.

Funding Funding for this project was provided by the British Society for Rheumatology (BSR). The BSR commissioned the Biologics Register (BSRBR) as a UK-wide national project to investigate the safety of biological agents in routine medical practice. DPMS and KLH are principal investigators on the BSRBR. BSR receives restricted income from UK pharmaceutical companies, presently Abbott Laboratories, Swedish Orphan Biovitrum, Merck, Pfizer, Roche and UCB Pharma. This income finances a wholly separate contract between the BSR and the University of Manchester. The principal investigators and their team have full academic freedom and are able to work independently of pharmaceutical industry influence. All decisions concerning analyses, interpretation and publication are made autonomously of any industrial contribution. Members of the Manchester team, BSR trustees, committee members and staff complete an annual declaration in relation to conflicts of interest. LKM is funded by a clinical research training fellowship from the Medical Research Council. ACG is partly supported by a fellowship from the Medical Research Council (no 89912).

The British Society for Rheumatology Biologics Register Control Centre Consortium consists of the following institutions (all in the UK): Antrim Area Hospital, Antrim (Dr Nicola Maiden); Cannock Chase Hospital, Cannock Chase (Dr Tom Price); Christchurch Hospital, Christchurch (Dr Neil Hopkinson); Royal Derby Hospital, Derby (Dr Sheila O’Reilly); Dewsbury and District Hospital, Dewsbury (Dr Lesley Hordon); Freeman Hospital, Newcastle-upon-Tyne (Dr lan Griffiths); Gartnavel General Hospital, Glasgow (Dr Duncan Porter); Glasgow Royal Infirmary, Glasgow (Professor Hilary Capell); Haywood Hospital, Stoke-on-Trent (Dr Andy Hassell); Hope Hospital, Salford (Dr Romela Benitha); King's College Hospital, London (Dr Ernest Choy); Kings Mill Centre, Sutton-in-Ashfield (Dr David Walsh): Leeds General Infirmary, Leeds (Professor Paul Emery); Macclesfield District General Hospital, Macclesfield (Dr Susan Knight); Manchester Royal Infirmary, Manchester (Professor lan Bruce); Musgrave Park Hospital, Belfast (Dr Allister Taggart); Norfolk and Norwich University Hospital, Norwich (Professor David Scott): Poole General Hospital, Poole (Dr Paul Thompson); Queen Alexandra Hospital, Portsmouth (Dr Fiona McCrae); Royal Glamorgan Hospital, Glamorgan (Dr Rhian Goodfellow); Russells Hall Hospital, Dudley (Professor George Kitas); Selly Oak Hospital, Selly Oak (Dr Ronald Jubb); St Helens Hospital, St Helens (Dr Rikki Abernethy); Weston General Hospital, Weston-super-Mare (Dr Shane Clarke/Dr Sandra Green); Withington Hospital, Manchester (Dr Paul Sanders); Withybush General Hospital, Haverfordwest (Dr Amanda Coulson); North Manchester General Hospital (Dr Bev Harrison); Royal Lancaster Infirmary (Dr Marwan Bukhari) and the Royal Oldham Hospital (Dr Peter Klimiuk).

\section{Competing interests None.}

Ethics approval The study was approved by the North-West Multicentre Research Ethics Committee.

Patient consent Obtained

Provenance and peer review Not commissioned; externally peer reviewed.

\section{REFERENCES}

1. McGuire JF, Ge NN, Dyson S. Nonmelanoma skin cancer of the head and neck l: histopathology and clinical behavior. Am J Otolaryngol 2009;30:121-33.

2. Cancerstats. Skin cancer. Cancer Research, UK. http://info.cancerresearchuk.org/ cancerstats/types/skin/ (accessed 29 June 2009).

3. Centers for Disease Control and Prevention. Skin cancer. http://www.cdc.gov/cancer/ skin/ (accessed 4 Oct 2011).

4. Karagas MR, Greenberg ER, Spencer SK, et al. Increase in incidence rates of basal cell and squamous cell skin cancer in New Hampshire, USA. New Hampshire Skin Cancer Study Group. Int J Cancer 1999;81:555-9.

5. Harris RB, Griffith K, Moon TE. Trends in the incidence of nonmelanoma skin cancers in southeastern Arizona, 1985-1996. J Am Acad Dermatol 2001;45:528-36.

6. Gridley G, McLaughlin JK, Ekbom A, et al. Incidence of cancer among patients with rheumatoid arthritis. J Natl Cancer Inst 1993:85:307-11.

7. Mellemkjaer L, Linet MS, Gridley G, et al. Rheumatoid arthritis and cancer risk. Eur J Cancer 1996;32A:1753-7.

8. Thomas $\mathbf{E}$, Brewster $\mathrm{DH}$, Black RJ, et al. Risk of malignancy among patients with rheumatic conditions. Int J Cancer 2000;88:497-502.

9. Esser AC, Abril A, Fayne S, et al. Acute development of multiple keratoacanthomas and squamous cell carcinomas after treatment with infliximab. J Am Acad Dermatol 2004;50(5 Suppl):S75-7

10. Askling J, Fored CM, Brandt L, et al. Risks of solid cancers in patients with rheumatoid arthritis and after treatment with tumour necrosis factor antagonists. Ann Rheum Dis 2005:64:1421-6.

11. Guidance on the use of etanercept and infliximab for the treatment of rheumatoid arthritis. National Institute for Health and Clinical Excellence. http://guidance.nice.org. uk/TA36 (accessed 15 Oct 2008).

12. Askling J, Fahrbach $\mathrm{K}$, Nordstrom B, et al. Cancer risk with tumour necrosis factor alpha (TNF) inhibitors: meta-analysis of randomized controlled trials of asalimumab, etanercept, and infliximab using patient level data. Pharmacoepidemiology \& Drug Safety 2010;19:1-11.

13. Wolfe F, Michaud K. Biologic treatment of rheumatoid arthritis and the risk of malignancy: analyses from a large US observational study. Arthritis Rheum 2007:56:2886-95

14. Amari W, Zeringue AL, McDonald JR, et al. Risk of non-melanoma skin cancer in a national cohort of veterans with rheumatoid arthritis. Rheumatology (Oxford) 2011;50:1431-9.

15. Ledingham J, Deighton C. Update on the British Society for Rheumatology guidelines for prescribing TNFalpha blockers in adults with rheumatoid arthritis (update of previous guidelines of April 2001). Rheumatology (Oxford) 2005:44:157-63.

16. Watson K, Symmons D, Griffiths I, et al. The British Society for Rheumatology biologics register. Ann Rheum Dis 2005;64 Suppl 4:iv42-3.

17. Kirwan JR, Reeback JS. Stanford Health Assessment Questionnaire modified to asses disability in British patients with rheumatoid arthritis. Br J Rheumatol 1986;25:206-9

18. Dickinson HO, Salotti JA, Birch PJ, et al. How complete and accurate are cancer registrations notified by the National Health Service Central Register for England and Wales? J Epidemiol Community Health 2001:55:414-22.

19. Goodwin RG, Holme SA, Roberts DL. Variations in registration of skin cancer in the United Kingdom. Clin Exp Dermatol 2004;29:328-30.

20. Clouser MC, Roe DJ, Foote JA, et al. Effect of non-steroidal anti-inflammatory drugs on non-melanoma skin cancer incidence in the SKICAP-AK trial. Pharmacoepidemiol Drug Saf 2009;18:276-83.

21. Holme SA, Malinovszky K, Roberts DL. Changing trends in non-melanoma skin cance in South Wales, 1988-98. Br J Dermatol 2000:143:1224-9.

22. 5-Year summary of incidence of non-melanoma skin cancer, basal cell carcinoma and squamous cell carcinoma in Scotland 2003-2007. http://www.isdscotland.org/ isd/1048.html. ISD Scotland, (accessed 07 Sept 2010).

23. Isomäki HA, Hakulinen T, Joutsenlahti U. Excess risk of lymphomas, leukemia and myeloma in patients with rheumatoid arthritis. J Chronic Dis 1978;31:691-6.

24. Cibere J Sibley J, Haga M. Rheumatoid arthritis and the risk of malignancy. Arthritis Rheum 1997;40:1580-6.

25. Hemminki K, Li X, Sundquist K, et al. Cancer risk in hospitalized rheumatoid arthritis patients. Rheumatology (Oxford) 2008;47:698-701.

26. Office for National Statistics. Cancer Statistics: Registrations Series MB1 - No. 39, 2008. Type: Internet Communication. http://ons.gov.uk/ons/ (accessed 11 July 2011)

27. Leombruno JP, Einarson TR, Keystone EC. The safety of anti-tumour necrosis factor treatments in rheumatoid arthritis: meta and exposure-adjusted pooled analyses of serious adverse events. Ann Rheum Dis 2009;68:1136-45.

28. Mercer, Galloway, Lunt et al. The Influence of anti-TNF therapy upon incidence of non-melanoma skin cancer (NMSC) in patients with rheumatoid arthritis (RA): results from the BSR Biologics Register (BSRBR) (abstract). ACR/ARPH Scientific Meeting 2009. 2009;2062 17-21 October 2009, Philadelphia, Pensylvania, USA.

29. Office for National Statistics. Summary quality report for cancer registration statistics http://www.ons.gov.uk/about-statistics/methodology-and-quality/quality/qualinfo-economic-social-and-bus-stats/quality-reports-for-social-statistics/index.htm (accessed 30 March 2011)

30. Marghoob A, Kopf AW, Bart RS, et al. Risk of another basal cell carcinoma developing after treatment of a basal cell carcinoma. J Am Acad Dermatol 1993:28:22-8. 